\title{
MRI and the pathology of breast invasive micropapillary carcinoma
}

\author{
CHUN-HONG HAN ${ }^{1,2}$, WEI-GEN YAO ${ }^{2}$, JIE HE ${ }^{1}$, ZHI-BIN GAO $^{3}$ and HONG-JIE HU ${ }^{1}$ \\ ${ }^{1}$ Department of Radiology, Sir Run Run Shaw Hospital, Zhejiang University School of Medicine, Hangzhou, Zhejiang 310016; \\ Departments of ${ }^{2}$ Radiology and ${ }^{3}$ Pathology, The Affiliated Yangming Hospital of Ningbo University, \\ Yuyao People's Hospital, Yuyao, Zhejiang 315400, P.R. China
}

Received August 22, 2019; Accepted May 5, 2020

DOI: $10.3892 / 01.2020 .11848$

\begin{abstract}
Diagnosis of breast invasive micropapillary carcinoma (IMPC) before surgery is of great value for determining the optimal treatment strategy. The aim of the present study was to investigate the magnetic resonance imaging (MRI) and pathological features of IMPC. MRI features of IMPC were characterized in relation to the patients' clinicopathological features. Clinical manifestations, mammography results and/or MRI findings of patients with IMPC were retrospectively analyzed. Parameters included morphology, plain T2-weighted imaging (T2WI) signal intensity, the apparent diffusion coefficient (ADC), the internal enhancement mode, early enhancement rates and time-intensity curve (TIC) types during dynamic enhanced scanning. A total of 10 lesions were detected by MRI in eight patients, with one case having three lesions with the mean diameter of $34.44 \mathrm{~mm}$. In plain T2WI scanning, the lesions appeared inhomogeneous with a moderate or high signal intensity. When the b value was $800 \mathrm{sec} / \mathrm{mm}^{2}$, the average ADC value was $0.823 \pm 0.12 \times 10^{-3} \mathrm{~mm}^{2} / \mathrm{sec}$. A total of four cases exhibited mass-like enhancement, including an oval rim in one case (three lesions), irregular inhomogeneous enhancement in two cases and irregular uniform enhancement in one case. The margins were clear in one case (three lesions), irregular in two cases and spiculate in one case. Among the four cases with non-mass enhancement, the distribution was
\end{abstract}

Correspondence to: Dr Hong-Jie Hu, Department of Radiology, Sir Run Run Shaw Hospital, Zhejiang University School of Medicine, 3 Qingchun East Road, Jianggan, Hangzhou, Zhejiang 310016, P.R. China

E-mail: hongjiehu@zju.edu.cn; hanchqiuyu@163.com

Abbreviations: IMPC, invasive micropapillary carcinoma; WHO, World Health Organization; MRI, magnetic resonance imaging; ROI, region of interest; ADC, apparent diffusion coefficient; TIC, time-intensity curve; BI-RADS, Breast Imaging Reporting and Data System; DCIS, ductal carcinoma in situ; ER, estrogen receptor; PR, progesterone receptor; HER2, human epidermal growth factor receptor 2

Key words: breast tumor, IMPC, MRI focal in two cases, linear in one case and regional in one case, and the internal enhancement mode was cluster-like in one case, heterogeneous in one case and uniform in two cases. The average early enhancement rate was $116.96 \pm 45.26 \%$. TICs of type III were observed in all cases. In conclusion, MRI of IMPC demonstrated typical features of malignant tumors and lymphatic vessel infiltration, suggesting that MRI may exhibit guiding significance for the diagnosis and treatment planning of IMPC.

\section{Introduction}

Invasive micropapillary carcinoma (IMPC) is a type of mammary epithelial tumor that was added in the 2003 World Health Organization (WHO) classification (1). IMPC was first described by Siriaunkgul and Tavassoli and was reported to account for $0.7-3 \%$ of all breast cancer cases (2). The pathological morphology of IMPC is unique, and immunohistochemistry has demonstrated that the positive portion of the epithelial membrane antigen is located on the outside of the pseudopapillary neoplasm or glandular tube (3). Most cases of IMPC appear mixed with other pathological types of invasive breast carcinoma, most commonly with invasive ductal carcinoma (4).

Lymphatic invasion and lymph node metastasis are common in IMPC, with an incidence of nodal metastases of $24.9 \%$, leading to frequent recurrence and poor prognosis in patients, with a 5-year overall survival rate of $87.5 \%$ (5). Even when the proportion of micropapillary structures is $<10 \%$, the invasive ability of the cancer is significantly higher compared with that of the same pathological type of breast cancer without IMPC components $(6,7)$. Diagnosis of this cancer type before surgery is of great value for determining the optimal treatment strategy (6), including the choice of surgical methods and the follow-up treatment plan to improve the prognosis of patients.

To date, the majority of studies have focused on the pathological features and ultrasonographic findings of $\operatorname{IMPC}(8,9)$. In previous reports, most IMPCs presented as an irregular mass with a high density and a non-circumscribed margin on the mammography, and as an irregular spiculated mass on magnetic resonance imaging (MRI) (10-16). These are typical features of malignant breast cancers (17); however, they are of limited significance for the diagnosis of IMPC. In addition, 
only several MRI features were investigated in each study, and thus, the results did not provide enough information for clinical use (10-14). Therefore, the present retrospective study was performed to characterize the MRI and pathological features of IMPC for comprehensive preoperative assessment of IMPC cases.

\section{Patients and methods}

Ethics approval. The present study was approved by the Ethics Committee of Sir Run Run Shaw Hospital, Zhejiang University School of Medicine (Hangzhou, China). All procedures involving human participants were performed in accordance with the ethical standards of the Institutional and/or National Research Committee, the 1964 Helsinki Declaration and its later amendments or comparable ethical standards. Informed consent was waived due to the retrospective nature of the study.

Patients. A total of nine cases of IMPC, confirmed pathologically after surgical resection or ultrasound-guided core-needle breast biopsy at Sir Run Run Shaw Hospital, Zhejiang University School of Medicine between August 2011 and January 2018, were included in the present study. A total of six patients underwent both MRI and mammography, two patients underwent MRI only and one patient underwent mammography only. None of the patients had received radiotherapy or undergone biopsy before MRI examination, and complete immunohistochemical data were available for all lesions.

Imaging. Preoperative mammography was performed in seven cases using a GE Senographe DS digital mammography machine (GE Healthcare) in the routine craniocaudal and mediolateral oblique position.

Preoperative MRI was performed in eight cases with a GE Signa HD Excite 1.5T/HD or 3.0T superconducting MRI scanner (GE Healthcare), with an 8-channel breast phased array surface coil. Patients were in a prone position, and bilateral breasts were hanging naturally in the coil. The plain cross-sectional T2WI fat suppression sequence and fast spin-echo T2WI sequence were scanned at a layer thickness of $4.0 \mathrm{~mm}$ with a layer spacing of $1.0 \mathrm{~mm}$. The sagittal T2WI fat suppression sequence was scanned at a layer thickness of $4.0 / 5.0 \mathrm{~mm}$ with a layer spacing of $1.0 \mathrm{~mm}$, a matrix of $512 \times 512$ and number of excitations (NEX)2. Diffusion-weighted imaging was performed using single-shot plane echo-planar imaging technology with a matrix of $256 \times 256$, layer thickness of $4.0 \mathrm{~mm}$, layer spacing of $1.0 \mathrm{~mm}$, NEX6 and diffusion sensitivity coefficient b values of 0 and $800 \mathrm{sec} / \mathrm{mm}^{2}$.

Dynamic enhanced scanning was performed using breast-optimized parallel acquisition of 3D fast gradient echo sequences (volume imaging for breast assessment). The dynamic enhancement was performed before the masking, and $0.2 \mathrm{mmol} / \mathrm{kg}$ of the contrast agent Gd-DTPA was injected via a high-pressure syringe through the elbow vein at a rate of $3.0 \mathrm{ml} / \mathrm{sec}$, followed by the injection of $15-20 \mathrm{ml}$ saline. Scanning was performed immediately. A total of seven phases were continuously collected, and the scanning time per phase was $60 \mathrm{sec}$. Imaging was performed with a reverse angle of $15^{\circ}$, a matrix of $512 \times 512$, and NEX2. All patients' dynamic enhanced scans were post-processed on the GE
AW4.5 workstation using Functool software (both from GE Healthcare). On each scan, the region of interest (ROI) was selected in the most obvious area of lesion enhancement, avoiding the necrotic cystic region, and the TIC was plotted.

Pathological examination. All IMPC tissue specimens were fixed in $10 \%$ neutral formalin for $12 \mathrm{~h}$ at room temperature, embedded in paraffin and serially sectioned with a layer thickness of $4 \mu \mathrm{m}$. Subsequently, they were stained with hematoxylin for $5 \mathrm{~min}$ and eosin for $3 \mathrm{~min}$ at room temperature. Immunohistochemical staining was performed using the EnVision (cat. no. K5007; Dako; Agilent Technologies, Inc.) method. Briefly, 4\% goat serum (OriGene Technologies, Inc.) was added dropwise for blocking at room temperature for $30 \mathrm{~min}$. Subsequently, different primary antibodies, namely anti-estrogen receptor (ready to use; cat. no. 790-4325; Roche Diagnostics), anti-progesterone receptor (ready to use; cat. no. 790-4296; Roche Diagnostics), anti-human epidemic growth factor receptor 2 (ready to use; cat. no. 790-4493; Roche Diagnostics) and anti-Ki67 (1:800; cat. no. M7240; Dako; Agilent Technologies, Inc.), were added and incubated at room temperature for $2 \mathrm{~h}$. After washing with PBS, one drop of EnVision secondary antibody (ready to use; cat. no. K5007; Dako; Agilent Technologies, Inc.) bound to horseradish peroxidase was added at $37^{\circ} \mathrm{C}$ for $30 \mathrm{~min}$. All stained sections were observed under a light microscope (magnifications $\mathrm{x} 40$ and $\mathrm{x} 100$ ).

Image analysis. All mammography and MRI scans were reviewed by two physicians with $>5$ years of experience in breast lesion diagnosis, who were blinded to the pathological results. All signs were described in accordance with the 2013 version of the Breast Imaging Reporting and Data System (BI-RADS) proposed by the American College of Radiology (18). The features of mammography included lesion type (only mass, mass with suspected malignant calcification, only suspected malignant calcification, focal asymmetry or structural distortion) and mass characteristics (shape and edge). MRI analysis included determination of the lesion size, plain scanning signal intensity (low, equal and high signal compared with that in normal glands), enhanced lesion morphology, edge morphology, internal enhancement mode, early enhancement rate and the TIC. Lesion size was evaluated using the maximum diameter as the reference index. If multiple lesions were present, the diameter of the largest lesion was measured.

TICs were generated by the post-processing workstation. According to the 2013 BI-RADS, the enhancement rate was based on the first phase enhancement rate as follows: $\left(\mathrm{SI}_{\text {post }}-\mathrm{SI}_{\text {pre }}\right) / \mathrm{SI}_{\text {pre }} \times 100 \%$, where $\mathrm{SI}_{\text {post }}$ is the intensity of the first phase signal after lesion enhancement, and $\mathrm{SI}_{\text {pre }}$ is the signal intensity before enhanced scanning. The enhancement rate was classified into three modes: Slow $(<50 \%)$, medium $(50-100 \%)$ and fast $(>100 \%)$. The delay period was recorded after the appearance of the peak, with the progressive type recorded as type I, the platform type recorded as type II and the outflow type recorded as type III. For lesions with complicated or non-tumor enhancement, numerous points were repeatedly evaluated, and multiple TICs were considered together.

The ADC map was automatically generated by the workstation and used to artificially measure the ADC value. 
A visibly solid part of the lesion was selected, and the ROI was manually selected according to the size of the lesion. The ROI was usually drawn slightly smaller than the lesion range, avoiding the cystic change and hemorrhagic or necrotic areas. ROIs were measured 3-5 times for the calculation of the mean value.

All pathological sections were reviewed by two pathologists with 5 years of experience in the diagnosis of breast diseases, and agreement was achieved through discussion when the diagnoses were inconsistent. According to the 4th edition of the WHO classification of breast tumors published in 2012 (19), IMPC was identified by clusters of cells in a pseudo-papillary structure without a fibrous vascular axis that were surrounded by interstitial spaces. In addition, the examination included the presence of associated ductal carcinoma in situ (DCIS), lymphatic vessel infiltration, axillary lymph node status, proliferation index (Ki-67), and the expression of estrogen receptor (ER), progesterone receptor (PR) and human epidermal growth factor receptor 2 (HER2). Molecular subtype was determined based on ER, PR, HER2 and Ki-67 expression and categorized as follows: Luminal A was $\mathrm{ER}^{+}$and/or $\mathrm{PR}^{+}, \mathrm{HER}^{-}$and $\mathrm{Ki}-67^{-}$; luminal $\mathrm{B}^{-}$was $\mathrm{ER}^{+}$ and/or $\mathrm{PR}^{+}$, HER2- and $\mathrm{Ki}^{-} 67^{+}$; luminal-HER2-positive was $\mathrm{ER}^{+}$and/or $\mathrm{PR}^{+}$and HER2 ${ }^{+}$; HER2-rich was ER ${ }^{-}, \mathrm{PR}^{-}$and $\mathrm{HER}^{+}$; and triple negative was $\mathrm{ER}^{-}, \mathrm{PR}^{-}$and HER2-

\section{Results}

Clinical characteristics. All nine patients were female with an average age of 52.11 years (range, 40-65 years). Among them, seven patients were postmenopausal. The initial manifestations were a palpable breast mass in $8(89 \%)$ patients and a gradually enlarged breast mass in two patients. Three patients reported mild tenderness of the mass, and two patients reported ipsilateral breast pain. Eight (80\%) lesions were located in the left breast. The mean lesion diameter was $34.44 \pm 25.68 \mathrm{~mm}$, and the range between the minimum and maximum diameter was 13.2-85.4 $\mathrm{mm}$, with a median value of $18.3 \mathrm{~mm}$. The patient clinical characteristics are shown in Table I.

Pathological manifestations. Regarding the pathological manifestations of the nine cases, there were three cases accompanied by DCIS, seven cases with lymph node metastasis (including one case of lymph node metastasis in the supraclavicular region), three cases with lymphatic invasion, one case with vascular tumor thrombus, two cases with invasion of the nipple and one case with invasion of the pectoralis major. According to the immunohistochemical staining analysis, three cases were luminal $\mathrm{A}\left(\mathrm{ER}^{+}\right.$and $\left.\mathrm{PR}^{+}\right)$, four cases were luminal $\mathrm{B}$ (four cases were $\mathrm{ER}^{+}$and three cases were $\mathrm{PR}^{+}$), and two cases were luminal-HER2-positive $\left(\mathrm{ER}^{+}, \mathrm{PR}^{+}, \mathrm{HER} 2^{+}\right.$and Ki-67+) (Table I).

\section{Imaging}

Mammography features. A total of seven patients underwent mammography before surgery, and internal mammary gland lesions were identified in six patients. A total of five cases exhibited high-density masses with an oval or irregular shape and blurring or spiculation and partially visible lobes. Among them, two cases had small dense or polymorphic suspicious malignant calcifications with segmental distribution, including one case with calcification around the mass; two cases exhibited local skin thickening or invagination; and one case had multiple enlarged axillary lymph nodes with disappearance of the hilum of the lymph nodes. One case presented with structural asymmetry, small pleomorphic calcification with regional distribution, localized skin thickening, nipple retraction, multiple lymph nodes with axillary fossa and disappearance of the hilum. No abnormalities were observed in the mammary gland of one patient; instead, only enlarged axillary lymph nodes were detected (Table II).

MRI. On plain T2WI, seven cases had slightly high heterogeneous signal and one had a high signal. With a $b$ value of $800 \mathrm{sec} / \mathrm{mm}^{2}$, the average, maximum, minimum and median ADC values were $0.823 \pm 0.12 \times 10^{-3}, 0.989 \times 10^{-3}, 0.613 \times 10^{-3}$ and $0.844 \times 10^{-3} \mathrm{~mm}^{2} / \mathrm{sec}$, respectively. In the enhanced scanning, four cases exhibited mass-like enhancement, including one case (three lesions) with oval-shaped ring enhancement, one case with irregular shape heterogeneity enhancement and one case with irregular shape uniform enhancement. The margins were clear in one case (three lesions), irregular in two cases and spiculated in one case (Fig. 1). A total of four cases exhibited non-mass enhancement, including two cases with a focal distribution, one case with linear distribution and one case with regional distribution. Regarding the internal enhancement, these four cases included one case with clustering, one case with heterogeneity and two cases with uniformity (Fig. 2). The average, maximum, minimum and median early enhancement rates were 116.96 $\pm 45.26,190.1,20.3$ and $126.1 \%$, respectively. TICs were of type III in all cases. IMPC was accompanied by skin edema thickening in one case, local skin depression in one case and nipple depression in one case. Axillary lymphadenopathy with enhancement was observed in three cases. The sensitivity, specificity, positive predictive value, negative predictive value and overall accuracy of MRI for axillary lymph node metastasis diagnosis were 50, 100, 100,40 and $62.5 \%$, respectively (Table III).

\section{Discussion}

IMPC is a highly invasive type of breast cancer (20). The incidence of IMPC is very low accounting for $0.76-3.8 \%$ of breast carcinomas (21-23); however, the degree of malignancy is high. In the present study, the clinical manifestations, pathological changes and imaging results in cases of IMPC were investigated, including mammography and MRI findings. The most common clinical manifestations were palpable masses that were presented in $89 \%$ (8/9) of the cases studied, and this percentage was slightly lower than compared with the $94 \%$ reported by Günhan-Bilgen et al (17). Among the observed lesions, the site with the most frequent IMPC occurrence was the left breast $(80 \%, 8 / 10)$, which was consistent with the finding of $60.5 \%$ of lesions $(23 / 38)$ in the left breast reported by Kim et al (24). In most cases, axillary lymph node metastasis was present at the time of diagnosis, and axillary lymphadenopathy was noticed before the primary tumor was identified in one case of the present study.

The mammography results of all patients in the present study suggested malignant tumors, and the characteristics 


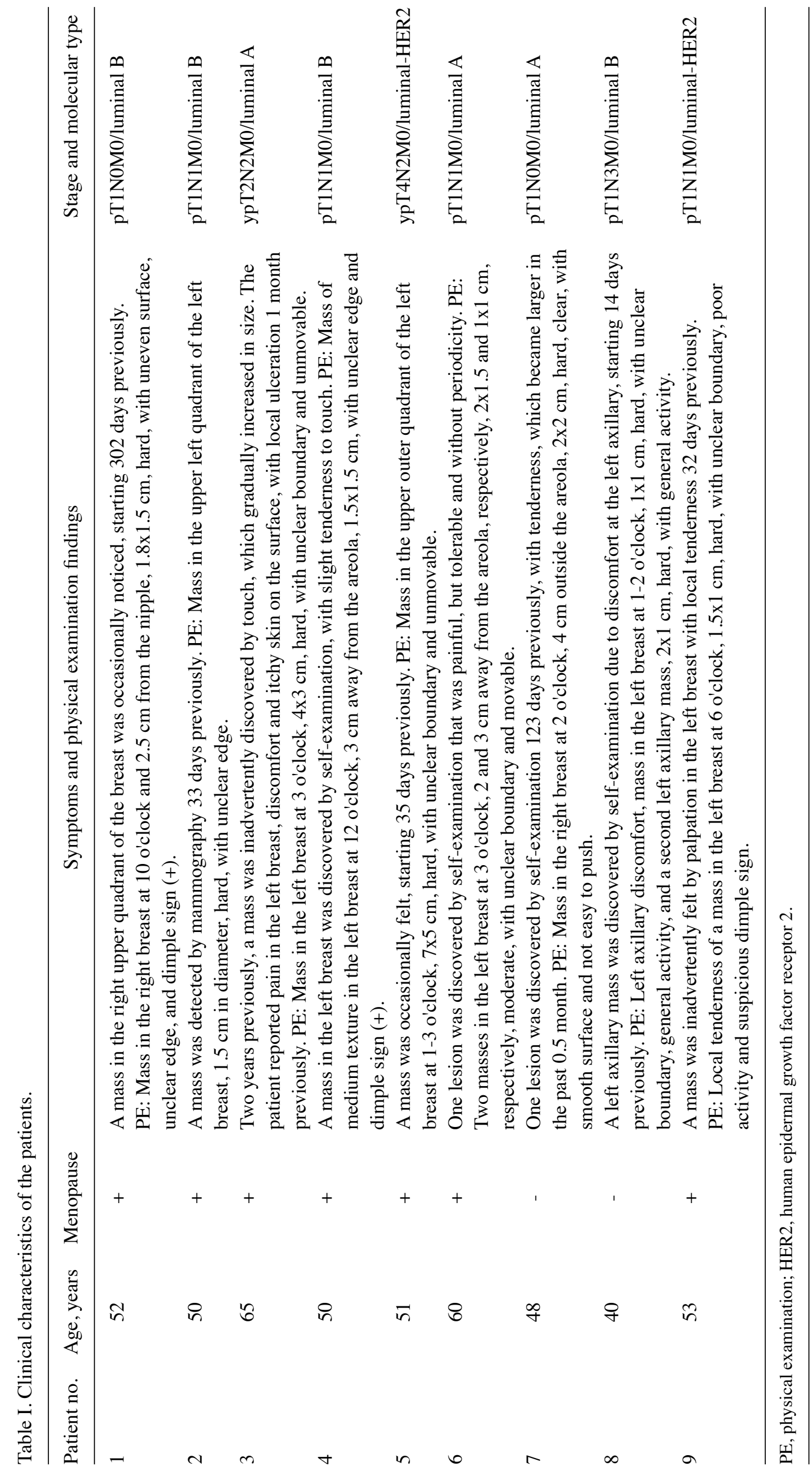




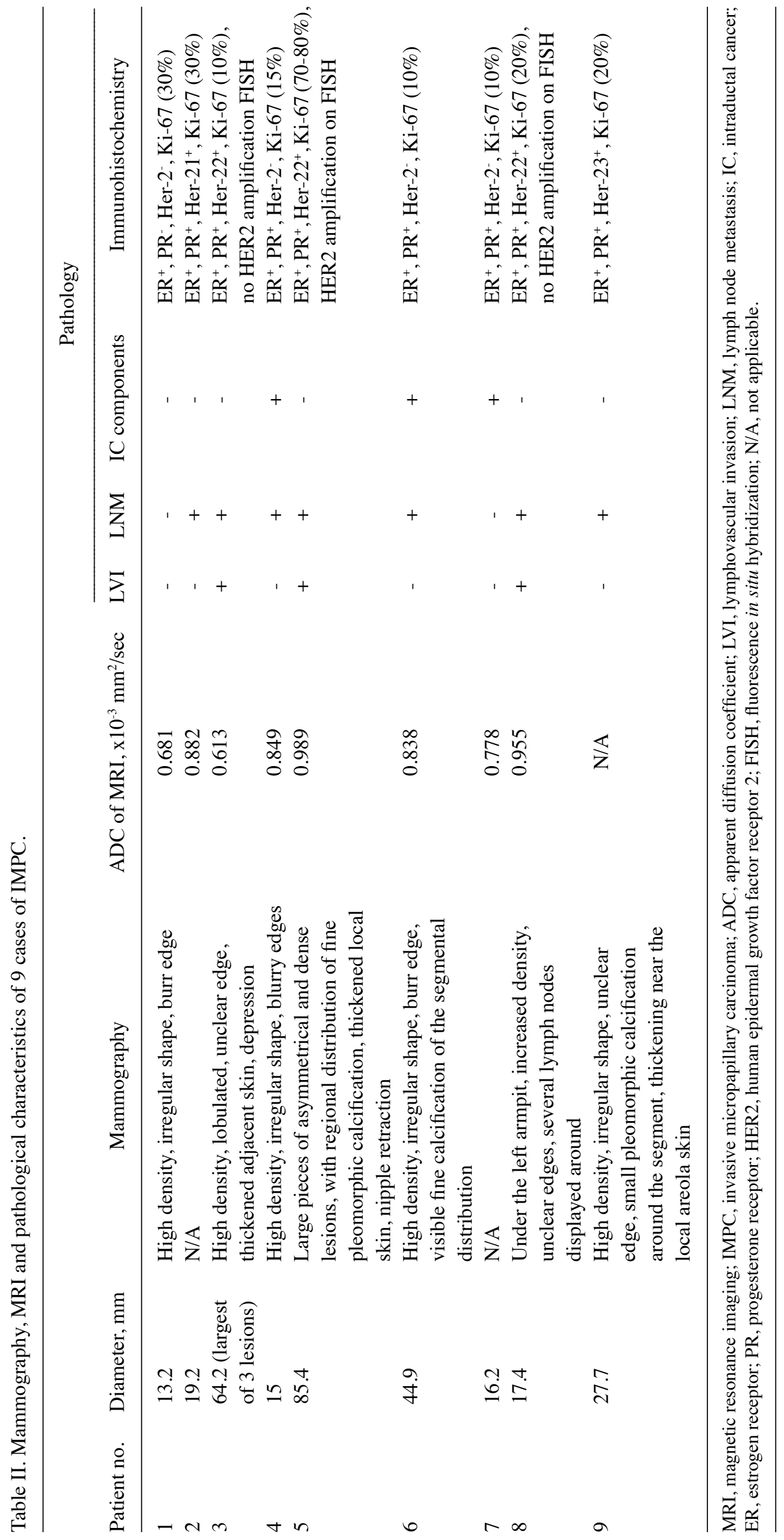



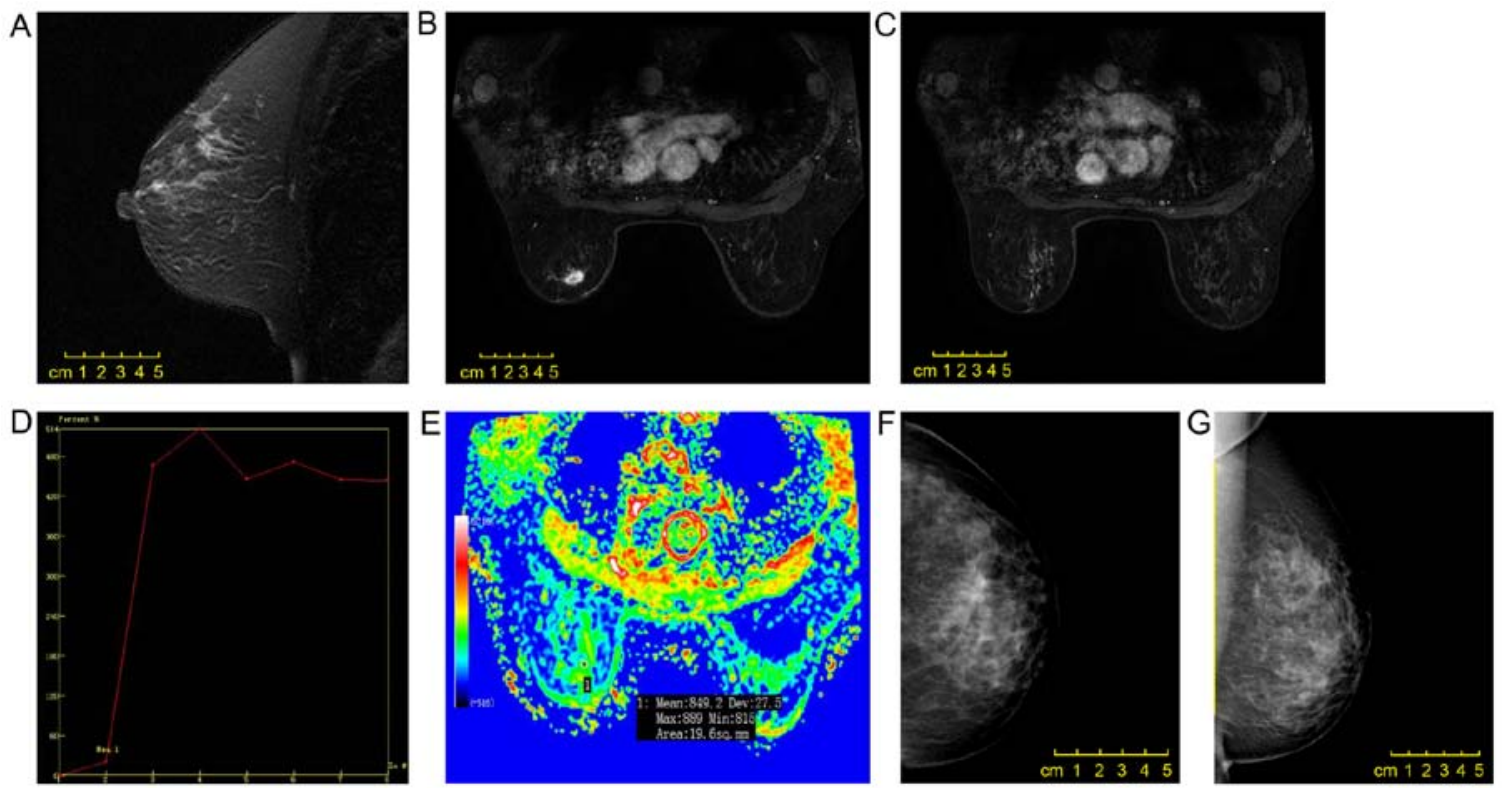

Figure 1. Scans from a 50-year-old female patient. (A) A mass with irregular shape, (B) spiculation and (C) heterogeneous enhancement was observed in the left upper quadrant of the breast by enhanced magnetic resonance imaging. (D) The early enhancement rate was $20.3 \%$, and the time-intensity curve was of type III. (E) The observed diffusion coefficient value was $0.849 \times 10^{-3} \mathrm{~mm}^{2} / \mathrm{sec}$. The invasive micropapillary carcinoma was surrounded by a non-mass enhancement lesion with segmental distribution and an internal cluster-like enhanced region, which was pathologically confirmed as simultaneous ductal carcinoma in situ. (F and G) Mammography revealed a slightly dense mass with an irregular shape and spiculation.
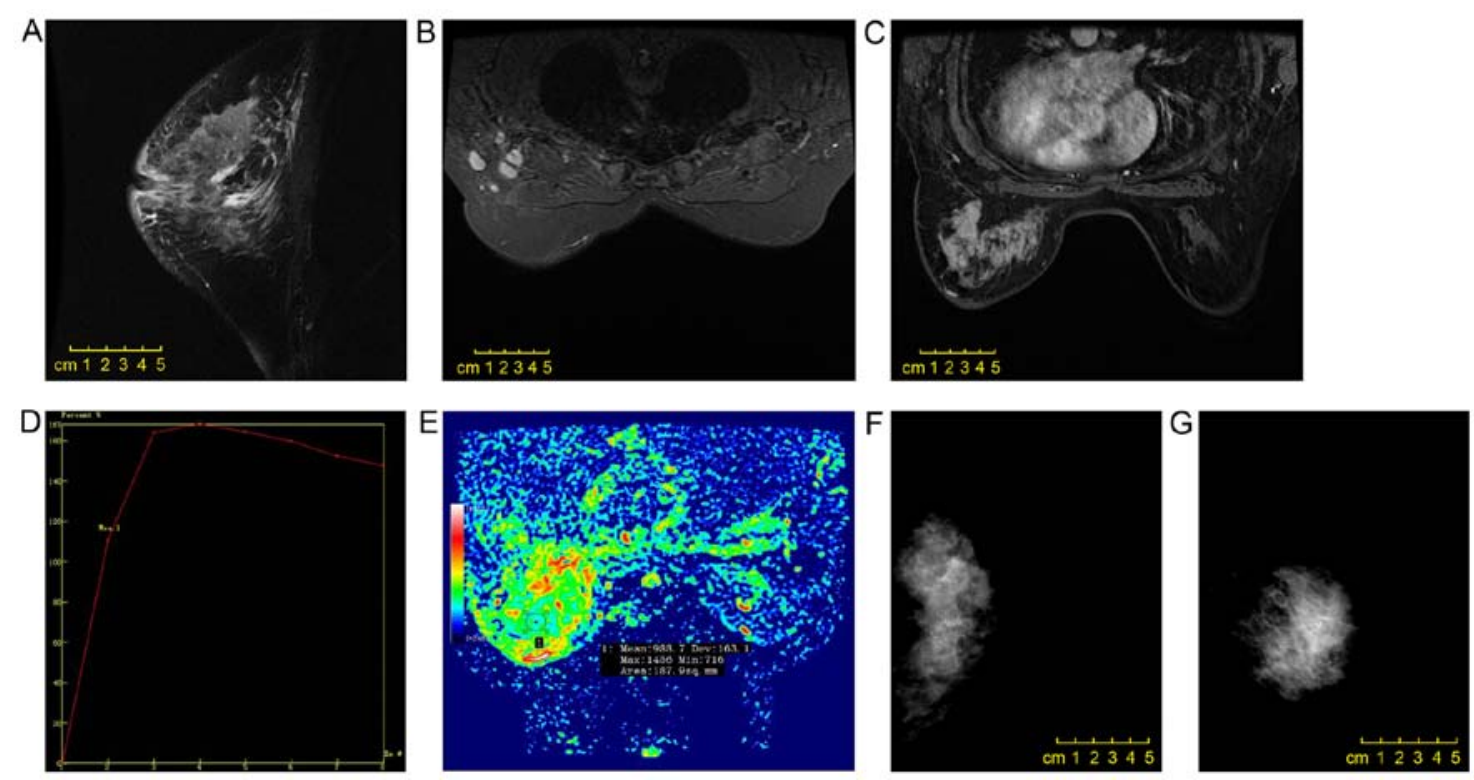

Figure 2. Scans from a 51-year-old female patient. (A) A non-mass enhancement lesion with (B) a regional distribution and (C) an internal cluster-like enhancement was observed in the left upper quadrant of the breast by enhanced magnetic resonance imaging. (D) The early enhancement rate was $110.3 \%$, and the time-intensity curve was of type III. (E) The apparent diffusion coefficient value was $0.989 \times 10^{-3} \mathrm{~mm}^{2} / \mathrm{sec}$, and invasive micropapillary carcinoma was accompanied by (A) thickening of the left areola skin, nipple retraction, and (B) multiple lymph nodes in the left axillary fossa. (F and G) Mammography revealed a large mass with asymmetric density in the left breast, extending beyond one quadrant, with a regional distribution of small polymorphic calcification, local skin thickening, nipple retraction and axillary lymph node enlargement.

were mostly consistent with those observed in the literature (17,25-28), including a mass (54.2-74.4\%), irregular high density and microcalcification (38.5-66.7\%). In the study by Lim et al (13), microcalcification was observed around the edge of the mass. As reported be Adrada et al (10) and Alsharif et al (12), the common morphological features of microcalcification were polymorphism $(57 \%, 11 / 19)$ and polymorphism or fine branch morphology $(86.7 \%, 13 / 15)$.
Microcalcification is the most common mammographic feature of DCIS (29), and these imaging features are highly suggestive of malignant tumors.

Previous studies have reported that IMPC exhibits an irregular mass with an irregular or spiculated edge on MRI $(10,13)$. The enhanced scanning curve is outflow-type and the internal enhancement mode is very different, with $16.7-38.9 \%$ probability of surrounding non-mass-enhanced lesions (10-14). 
Table III. MRI characteristics of pure breast IMPC.

\begin{tabular}{|c|c|}
\hline Features & $\mathrm{n}(\%)$ \\
\hline Mass-like enhancement & 4 \\
\hline \multicolumn{2}{|l|}{ Shape } \\
\hline Oval & $1(25)$ \\
\hline Irregular shape & $3(75)$ \\
\hline \multicolumn{2}{|l|}{ Edge } \\
\hline Clear & $1(25)$ \\
\hline Irregular & $2(50)$ \\
\hline Burr & $1(25)$ \\
\hline \multicolumn{2}{|l|}{ Internal enhancement feature } \\
\hline Uniform & $1(25)$ \\
\hline Inhomogeneous & $2(50)$ \\
\hline Edge enhancement & $1(25)$ \\
\hline Non-mass enhancement & 4 \\
\hline \multicolumn{2}{|l|}{ Distribution } \\
\hline Linear & $1(25)$ \\
\hline Focal & $2(50)$ \\
\hline Regional & $1(25)$ \\
\hline \multicolumn{2}{|l|}{ Internal enhancement feature } \\
\hline Uniform & $2(50)$ \\
\hline Inhomogeneous & $1(25)$ \\
\hline Cluster-like & $1(25)$ \\
\hline TIC & 8 \\
\hline \multicolumn{2}{|l|}{ Early stage } \\
\hline Slow & $1(12.5)$ \\
\hline Medium & $1(12.5)$ \\
\hline Fast & $6(75)$ \\
\hline \multicolumn{2}{|l|}{ Delay stage } \\
\hline Outflow type & $8(100)$ \\
\hline Axillary lymph node metastasis & 6 \\
\hline$(+)$ on MRI & $3(50)$ \\
\hline (-) on MRI & $3(50)$ \\
\hline Metastatic lymph nodes detected by MRI & 8 \\
\hline Sensitivity & $50 \%$ \\
\hline Specificity & $100 \%$ \\
\hline Positive predictive value & $100 \%$ \\
\hline Negative predictive value & $40 \%$ \\
\hline Overall accuracy & $62.5 \%$ \\
\hline Invasion of the nipple, chest wall or skin & $2(25)$ \\
\hline
\end{tabular}

MRI, magnetic resonance imaging; IMPC, invasive micropapillary carcinoma; TIC, time-intensity curve.

Secondly, 22-39\% of cases presented with non-mass enhancement in these studies (10-14). Kubota et al (15) compared the MRI features of eight IMPC lesions and 22 invasive ductal carcinomas and demonstrated that IMPC was more likely to exhibit a characteristic irregular mass. In the present study, $50 \%$ of IMPC cases exhibited mass-like enhancement, and the other $50 \%$ exhibited non-mass enhancement. The incidence of non-mass enhancement was higher compared with that reported in the literature, which was possibly due to the increase of the pathological micro-nipple component. This needs to be confirmed by analysis of more cases. Among the four cases of mass-like enhanced lesions and the four cases of non-mass enhanced lesions, one case in each set had non-mass enhancement of segmental distribution, which was pathologically confirmed to be accompanied by DCIS. In the four lesions with non-mass enhancement, two cases had a focal distribution, one case had a linear distribution and one case had a regional distribution, which differed from previous reports. Yun et al (11) reported seven cases with segmental distribution, and Jones et al (14) reported that most lesions with non-mass enhancement had a diffuse distribution. The internal enhancement patterns in the present study also differed, and TICs were of type III in all cases, which was consistent with a previous study (14). In addition, the ADC with a b value of $800 \mathrm{sec} / \mathrm{mm}^{2}$ was determined, and a mean ADC value of $0.823 \pm 0.12 \times 10^{-3} \mathrm{~mm}^{2} / \mathrm{sec}$ was observed, which indicated malignant tumors (16). The enhancement rate in the first phase was mostly the rapid enhancement mode $(75 \%, 6 / 8)$ according to the 2013 version of the BI-RADS (19), and the average was $116.96 \pm 45.26 \%$. Although the standard deviation for the mean enhancement rate was large, the early rapid enhancement mode also suggested the differentiation of malignant tumors. MRI demonstrated higher sensitivity for lesion detection compared with that of mammography, especially for the detection of lesions with non-mass enhancement and for precise definition of the lesion $(13,14)$. Kubota et al $(15)$ concluded that radiography, including $\mathrm{MRI}$, can be carefully interpreted to determine the boundary of the lesion, and additional resection can be performed at the positive edge (cells close to the edge of the previously resected specimen).

Previous studies have reported that IMPC is often associated with DCIS, with an incidence as high as $78.6 \%(10,13,25)$. Among the patients in the present study, only three cases (33\%) had IMPC mixed with DCIS, and the incidence was lower than previously reported. The rate of axillary lymph node metastasis in IMPC has been reported to range between 56 and $90.5 \%(12-14,26,27)$, and lymphatic vascular invasion has been demonstrated to be an independent factor for poor prognosis and a marker of lymph node metastasis $(4,24)$. Zekioglu et al (4) observed lymphatic invasion in $75 \%$ of cases of IMPC, of which $82 \%$ exhibited lymph node metastasis. In the present study, lymphatic invasion was identified in three cases $(33.3 \%, 3 / 9)$, whereas axillary lymph node metastasis was observed in seven cases $(77.8 \%, 7 / 9)$. The longest lesion diameter in a case with lymphatic infiltration and simultaneous intravascular tumor thrombus lesions was $17.4 \mathrm{~mm}$, and the initial clinical manifestation was palpable axillary lymphadenopathy, indicating that the size of IMPC was not directly related to tumor invasion and histological characteristics, including histological grade; thus, lymphatic vessel density and lymphocytic infiltration of IMPC may be more relevant than lesion size (28). MRI revealed that three cases $(37.5 \%$, $3 / 8$ ) had axillary lymph node metastasis. The sensitivity, specificity, positive predictive value, negative predictive value and overall accuracy of MRI for axillary lymph node metastasis diagnosis were $50,100,100,40$ and $62.5 \%$, respectively. These results suggested that the possibility of axillary lymph node metastasis in patients with IMPC was high regardless of 
the MRI findings, and pathological examination of lymphatic vessels and regional lymph nodes was needed.

The molecular phenotype of lesions in the present study was mainly luminal type $(78 \%, 7 / 9)$, and no cases were triple negative, which was similar to the findings presented in previous reports $(4,11)$. Positivity for hormone receptors is usually observed in better differentiated tumors, and the prognosis is better in these cases. This seems to contrast with the high rates of biological invasion and recurrence and poor prognosis in IMPC. It is speculated that IMPC has unique histological features. In the present study, 2 cases $(22 \%, 2 / 9)$ were luminal-HER2 positive, and this frequency of HER2-positive cases was lower than those previously reported $(4,10,11)$. Previous studies have confirmed that HER2 gene amplification and/or upregulation of HER2 protein expression is associated with poor prognosis in patients with invasive breast cancer $(26,30)$. In the present study, one patient with the luminal-HER2-positive type presented with metastasis to the ipsilateral chest wall 2 years after treatment with chemoradiotherapy, modified radical mastectomy and endocrine therapy. After changing the chemotherapy regimen, multiple liver, lung and contralateral breast cancer metastases were observed. Retrospective analysis of the molecular phenotype in this case, in addition to HER2 $2^{2+}$ and HER2 gene amplification by fluorescence in situ hybridization and $\mathrm{Ki}-67$ index measurement ( $70-80 \%$, indicating a high cell proliferation index), indicated accelerated tumor metastasis. In the present study, the negative predictive value of MRI for axillary lymph node metastasis was only $40 \%$, indicating that regardless of the clinical and imaging findings for lymph nodes, patients with IMPC should undergo biopsy of the axillary lymph nodes.

Complete surgical resection of the lesion is an important therapy in IMPC (5). However, IMPC is typically irregular in shape on MRI and the border is unclear $(8,10)$. Thus, the possibility of residual cancer cells remaining in the breast after breast-conserving surgery is high. If a rapid pathological analysis performed during surgery reveals the presence of cancer cells at the margin of the resected specimen, re-expansion of the resection is required, and a prolonged operative time and re-expanded resection increase the probability of postoperative complications.

The present study had several limitations. First, the sample size was small, and more cases are needed. Second, the study was a retrospective analysis, and not all patients underwent both mammography and MRI examinations. Third, pathology was not classified according to the WHO guidelines. A multicenter study is the next step to expand the sample size and compare the MRI features, pathological grades and molecular phenotypes to identify effective imaging features for preoperative evaluation and prognosis prediction.

In conclusion, the MRI features of IMPC included typical malignant tumor characteristics with ready invasion of lymphatic vessels. Among the IMPC cases presented, frequent nodal metastases and high likelihood of luminal type lesions were observed. These characteristics provide valuable insight for the diagnosis of IMPC.

\section{Acknowledgements}

Not applicable.

\section{Funding}

No funding was received.

\section{Availability of data and materials}

The datasets used and/or analyzed during the current study are available from the corresponding author on reasonable request.

\section{Authors' contributions}

$\mathrm{CHH}$ and HJH designed/performed the majority of the experiments and data analysis, and wrote the manuscript. ZBG provided pathological assistance. WGY and JH contributed to the analysis and the interpretation of the data. All authors read and approved the final manuscript.

\section{Ethics approval and consent to participate}

The study was approved by the Ethics Committee of Sir Run Run Shaw Hospital, Zhejiang University School of Medicine (Hangzhou, China). All procedures involving human participants were performed in accordance with the ethical standards of the Institutional and/or National Research Committee, the 1964 Helsinki Declaration and its later amendments. All data published here are under the consent for publication.

\section{Patient consent for publication}

Not applicable.

\section{Competing interests}

The authors declare that they have no competing interests.

\section{References}

1. Tavassoli FA and Devilee P: Pathology and genetics of tumors of the breast and female genital organs. World Health Organization classification of tumors. IARC Press, 2003.

2. Siriaunkgul S and Tavassoli FA: Invasive micropapillary carcinoma of the breast. Mod Pathol 6: 660-662, 1993.

3. Luna-More S, Gonzalez B, Acedo C, Rodrigo I and Luna C: Invasive micropapillary carcinoma of the breast. A new special type of invasive mammary carcinoma. Pathol Res Pract 190: 668-674, 1994.

4. Zekioglu O, Erhan Y, Ciris M, Bayramoglu H and Ozdemir N: Invasive micropapillary carcinoma of the breast: High incidence of lymph node metastasis with extranodal extension and its immunohistochemical profile compared with invasive ductal carcinoma. Histopathology 44: 18-23, 2004.

5. Lewis GD, Xing Y, Haque W, Patel T, Schwartz M, Chen A, Farach A, Hatch S, Butler EB, Chang J and Teh BS: Prognosis of lymphotropic invasive micropapillary breast carcinoma analyzed by using data from the national cancer database. Cancer Commun (Lond) 39: 60, 2019.

6. Li W, Han Y, Wang C, Guo X, Shen B, Liu F, Jiang C, Li Y, Yang Y, Lang R, et al: Precise pathologic diagnosis and individualized treatment improve the outcomes of invasive micropapillary carcinoma of the breast: A 12-year prospective clinical study. Mod Pathol 31: 956-964, 2018.

7. Kaya C, Uçak R, Bozkurt E, Ömeroğlu S, Kartal K, Yazıcı P, Idiz UO and Mihmanli M: The impact of micropapillary component ratio on the prognosis of patients with invasive micropapillary breast carcinoma. J Invest Surg: 1-9, 2018. 
8. Kamitani K, Kamitani T, Ono M, Toyoshima S and Mitsuyama S: Ultrasonographic findings of invasive micropapillary carcinoma of the breast: Correlation between internal echogenicity and histological findings. Breast Cancer 19: 349-352, 2012.

9. Mizushima Y, Yamaguchi R, Yokoyama T, Ogo E and Nakashima O: Recurrence of invasive micropapillary carcinoma of the breast with different ultrasound features according to lesion site: Case report. Kurume Med J 58: 81-85, 2011.

10. Adrada B, Arribas E, Gilcrease $M$ and Yang WT: Invasive micropapillary carcinoma of the breast: Mammographic, sonographic, and MRI features. AJR Am J Roentgenol 193 W58-W63, 2009.

11. Yun SU, Choi BB, Shu KS, Kim SM, Seo YD, Lee JS and Chang ES: Imaging findings of invasive micropapillary carcinoma of the breast. J Breast Cancer 15: 57-64, 2012.

12. Alsharif S, Daghistani R, Kamberoğlu EA, Omeroglu A Meterissian S and Mesurolle B: Mammographic, sonographic and MR imaging features of invasive micropapillary breast cancer. Eur J Radiol 83: 1375-1380, 2014.

13. Lim HS, Kuzmiak CM, Jeong SI, Choi YR, Kim JW, Lee JS and Park MH: Invasive micropapillary carcinoma of the breast: MR imaging findings. Korean J Radiol 14: 551-558, 2013.

14. Jones KN, Guimaraes LS, Reynolds CA, Ghosh K, Degnim AC and Glazebrook KN: Invasive micropapillary carcinoma of the breast: Imaging features with clinical and pathologic correlation. AJR Am J Roentgenol 200: 689-695, 2013.

15. Kubota K, Ogawa Y, Nishioka A, Murata Y, Itoh S, Hamada N Morio K, Maeda $\mathrm{H}$ and Tanaka Y: Radiological imaging features of invasive micropapillary carcinoma of the breast and axillary lymph nodes. Oncol Rep 20: 1143-1147, 2008.

16. Peng YX, Cai HM and CY C: Vulation the differential value of diffusion-weighted imaging and dynamic contrast-enhanced magnetic resonance in breast lesions. Chinese Journal 11: 1-4, 2014.

17. Günhan-Bilgen I, Zekioglu O, Ustün EE, Memis A and Erhan Y: Invasive micropapillary carcinoma of the breast: Clinical, mammographic, and sonographic findings with histopathologic correlation. AJR Am J Roentgenol 179: 927-931, 2002.

18. Allarakha A, Gao Y, Jiang $\mathrm{H}$ and Wang PJ: Prediction and prognosis of biologically aggressive breast cancers by the combination of DWI/DCE-MRI and immunohistochemical tumor markers. Discov Med 27: 7-15, 2019.

19. American College of Radiology, Breast Imaging Reporting and Data System (BI-RADS). 4th Edition, American College of Radiology, Reston, 563-570, 2013.

20. Onder S, Fayda M, Karanlık H, Bayram A, Şen F, Cabioglu N, Tuzlalı S, İlhan R and Yavuz E: Loss of ARID1A expression is associated with poor prognosis in invasive micropapillary carcinomas of the breast: A clinicopathologic and immunohistochemical study with long-term survival analysis. Breast J 23 . 638-646, 2017.
21. Shi WB, Yang LJ, Hu X, Zhou J, Zhang Q and Shao ZM: Clinico-pathological features and prognosis of invasive micropapillary carcinoma compared to invasive ductal carcinoma: A population-based study from China. PLoS One 9: e101390, 2014.

22. Gokce H, Durak MG, Akin MM, Canda Y, Balci P, Ellidokuz H, Demirkan B, Gorken IB, Sevinc AI, Kocdor MA, et al: Invasive micropapillary carcinoma of the breast: A clinicopathologic study of 103 cases of an unusual and highly aggressive variant of breast carcinoma. Breast J 19: 374-381, 2013

23. Hashmi AA, Aijaz S, Mahboob R, Khan SM, Irfan M, Iftikhar N, Nisar M, Siddiqui M, Edhi MM, Faridi N and Khan A: Clinicopathologic features of invasive metaplastic and micropapillary breast carcinoma: Comparison with invasive ductal carcinoma of breast. BMC Res Notes 11: 531, 2018.

24. Kim MJ, Gong G, Joo HJ, Ahn SH and Ro JY: Immunohistochemical and clinicopathologic characteristics of invasive ductal carcinoma of breast with micropapillary carcinoma component. Arch Pathol Lab Med 129: 1277-1282, 2005.

25. Ross JS, Fletcher JA, Linette GP, Stec J, Clark E, Ayers M, Symmans WF, Pusztai L and Bloom KJ: The her-2/neu gene and protein in breast cancer 2003: Biomarker and target of therapy. Oncologist 8: 307-325, 2003.

26. Kuroda H, Sakamoto G, Ohnisi K and Itoyama S: Clinical and pathologic features of invasive micropapillary carcinoma. Breast Cancer 11: 169-174, 2004.

27. Nassar H, Wallis T, Andea A, Dey J, Adsay V and Visscher D: Clinicopathologic analysis of invasive micropapillary differentiation in breast carcinoma. Mod Pathol 14: 836-841, 2001.

28. Guo X, Chen L, Lang R, Fan Y, Zhang X and Fu L: Invasive micropapillary carcinoma of the breast: Association of pathologic features with lymph node metastasis. Am J Clin Pathol 126: 740-746, 2006.

29. Evans A, Pinder S, Wilson R, Sibbering M, Poller D, Elston C and Ellis I: Ductal carcinoma in situ of the breast: Correlation between mammographic and pathologic findings. AJR Am J Roentgenol 162: 1307-1311, 1994.

30. Ferretti G, Felici A, Papaldo P, Fabi A and Cognetti F: HER2/neu role in breast cancer: From a prognostic foe to a predictive friend. Curr Opin Obstet Gynecol 19: 56-62, 2007.

(7) $\odot$ This work is licensed under a Creative Commons Attribution-NonCommercial-NoDerivatives 4.0 International (CC BY-NC-ND 4.0) License. 